\title{
Chronic fatigue syndrome: a joint paediatric-psychiatric approach
}

\author{
Margaret I Vereker
}

Prolonged fatigue after an apparent viral infection, occurring sporadically or as an epidemic, has been described over the past 50 years. It has been given various names including Royal Free disease $^{1}$ and myalgic encephalomyelitis, but the preferred terms in the medical literature have been postviral fatigue syndrome ${ }^{2}$ or chronic fatigue syndrome (CFS). ${ }^{3}$ However, the validity of this syndrome as a nosological entity has created a good deal of controversy and remains in doubt. ${ }^{4}$

A constellation of symptoms make up the syndrome. There is fatigue of defined onset that is generally reported to follow a viral illness, often an influenza-like illness or an infection of the upper respiratory tract. The patient experiences profound fatigue with the initial illness and then fails to make the expected recovery, with fatigue that can persist over months or years. Fatigue is defined as a subjective sensation, which the patient often describes as tiredness or weariness and that occurs at rest. These patients also report a clear relationship of fatigue to activity. The term fatiguability has been used to describe the greater than normal fatigue that occurs after physical and sometimes after mental exertion in these patients. A great variety of associated symptoms have been described that include increased sleepiness, dizziness, vertigo, headache, difficulty in concentrating, sore throat, muscle weakness, and myalgia. The majority of patients have some emotional symptoms. There can be irritability and anxiety, tearfulness and depression. The fatigue and associated symptoms are of such severity as to impair significantly normal daily activities. There is a remarkable absence of physical signs and physical investigations fail to detect any organic pathology or current infection to account for the symptoms.

Two main virus groups have been implicated in the aetiology of the CFS: enteroviruses (particularly coxsackie B) and the herpes viruses. There is serological evidence that some cases may follow enterovirus infection, ${ }^{5}$ or infectious mononucleosis (Epstein-Barr virus). ${ }^{78}$ However, these findings have not established a specific association with the syndrome and the proposed causal relationship remains doubtful. ${ }^{9} 10$

Considerable psychiatric morbidity has been described in adult patients with CFS, ${ }^{11}$ but there has been little to date in the literature about this syndrome in childhood and no systematic research has been carried out.
However, an increasing number of children and adolescents appear to have been given the diagnosis in recent years. ${ }^{12}$ This has been a cause for concern ${ }^{13}$ because of the multiplicity of factors that probably contribute to this syndrome and because of the treatment implications which the diagnosis may carry. The syndrome can be associated with significant absence from school, restricted physical and social activity with some severe cases being housebound or bedbound for prolonged periods. The purpose of this article is to provide a clinical description of the characteristics and management of a series of consecutive cases which were seen by a paediatric liaison team from a child psychiatry department.

\section{Patient selection and assessment}

Ten patients were seen consecutively by the child psychiatry liaison team (CPLT), consisting of a consultant and senior registrar in child and adolescent psychiatry and a clinical psychologist, at Paxton House Family and Young Persons Unit, Reading, over a period of 12 months. Eight cases were referred by a paediatrician and two cases by a consultant in another specialty, at the Royal Berkshire and Battle Hospitals, Reading.

A working party with a consultant paediatrician, community paediatrician, consultant microbiologist, and the consultant and senior registrar in child and adolescent psychiatry of the CPLT met regularly to define criteria and draw up a protocol for the paediatric and psychiatric assessment and management of cases of CFS.

The following criteria were defined and all were fulfilled for inclusion in this series:

(1) A definable onset which may have been associated with a viral infection.

(2) Generalised fatigue

(3) A report of easy fatiguability.

(4) One of the following associated symptoms, headache, dizziness, moodiness, or peripheral sensory change.

(5) The symptoms were of sufficient severity to interfere significantly with normal social and school functioning.

(6) After appropriate investigation and consideration of the history by a paediatrician or other consultant there was no detectable organic pathology to account for the symptoms. 
Table 1 Patient characteristics

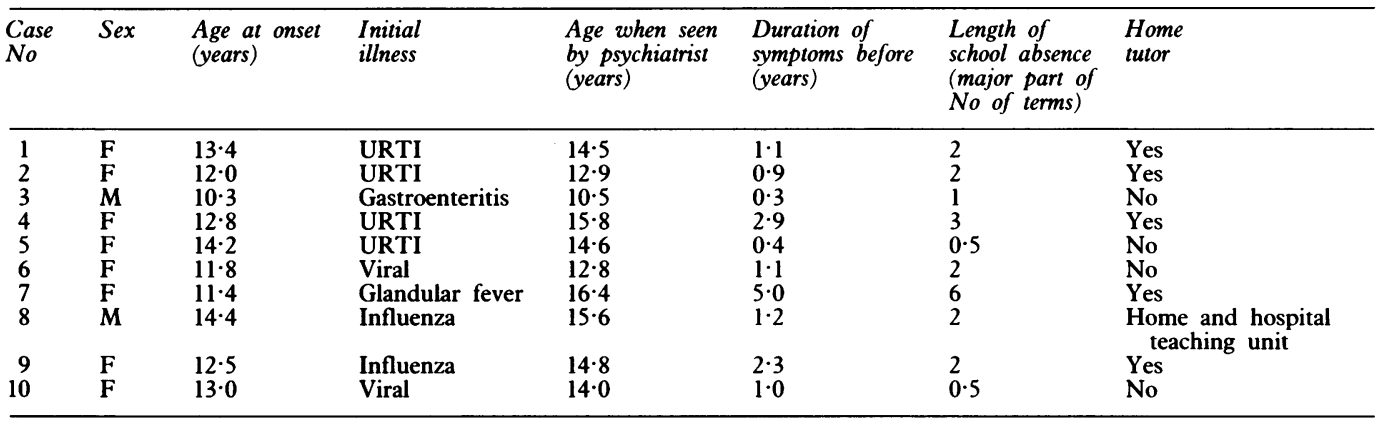

URTI=upper respiratory tract infection.

(7) Symptoms persisted at least six weeks after onset.

Where psychological elements were considered to be a major contributing factor a referral was made to the CPLT. A psychiatric assessment included individual and family interviews to identify psychiatric disorders and psychosocial factors which could be maintaining illness. The families in the series were remarkably reluctant to accept psychiatric involvement and where possible they were seen initially in a joint interview with the paediatrician or in a paediatric setting.

\section{Patient characteristics}

AGE AND GENDER

The children and adolescents in the series ranged in age from 10.5 years to 16.4 years (see table 1) when seen by a child psychiatrist. The mean (SD) age of onset was $12.6(1.3)$ years. The mean (SD) duration of symptoms before referral to a child psychiatrist was $1.6(1 \cdot 4)$ years.

There was a preponderance of girls with a female:male ratio of $4: 1$.

\section{PHYSICAL SYMPTOMS}

The patients experienced fatigue to a degree that interfered with their normal routine activities, and also prevented them from entering into leisure activities such as sports. Some found that if they did engage in strenuous activity, such as sports, they would feel increased generalised fatigue for days or weeks afterwards. For example, case 3 played football one afternoon scoring a goal, but was so exhausted after it that he missed the next three weeks of school.
Case 4 experienced such profound fatigue that she would spend half of the day in bed.

The patients had a range of associated symptoms, the most common of which were headache and dizziness. Two cases had headaches and one a sore throat of such severity that they required the daily administration of analgesics.

Four patients (see table 2) had symptoms that led to restrictions in physical mobility. This had a gradual onset with myalgia, muscle weakness, arthralgia, or joint stiffness leading to difficulty walking and an abnormal gait. Three of these patients were eventually unable to walk without support and were using a wheelchair, and two were completely bedbound. While bedbound case 7 experienced a sudden onset of total paralysis in her lower limbs. These three patients were so limited in their mobility that they were unable to perform general self care activities such as feeding, bathing, hair washing, and using the toilet unaided. They were admitted for investigation, including a neurological opinion. On examination these patients were found to be able to use muscle groups for one action that they were unable to use for another. The nursing staff observed that the loss of function was not consistent. For example posture and gait would vary with the onlookers and their relationship to the patient. The patient's symptoms or loss of function were often more severe if parents were present and least severe when they were unobserved.

\section{EMOTIONAL SYMPTOMS}

Two of the six patients with depressed mood had been treated by their general practitioner with antidepressants with no response. How-

Table 2 Symptoms

\begin{tabular}{|c|c|c|c|c|c|c|c|c|c|}
\hline \multirow{2}{*}{$\begin{array}{l}\text { Case } \\
\text { No }\end{array}$} & \multicolumn{4}{|c|}{ Symptoms causing physical restriction } & \multicolumn{5}{|c|}{ Emotional symptoms } \\
\hline & $\begin{array}{l}\text { Difficulty walking } \\
\text { and abnormal gait }\end{array}$ & $\begin{array}{l}\text { Using a } \\
\text { wheelchair }\end{array}$ & Bedbound & $\begin{array}{l}\text { Paralysis of } \\
\text { lower limbs }\end{array}$ & Depression & Anxiety & $\begin{array}{l}\text { Dissociative } \\
\text { symptoms }\end{array}$ & $\begin{array}{l}\text { Weight } \\
\text { loss }\end{array}$ & $\begin{array}{l}\text { Weight } \\
\text { gain }\end{array}$ \\
\hline 1 & + & - & - & - & + & - & - & + & - \\
\hline 2 & + & + & + & - & - & - & - & - & + \\
\hline 3 & - & - & - & - & + & - & - & - & + \\
\hline 4 & - & - & - & - & + & - & - & - & - \\
\hline 5 & - & - & - & - & - & + & - & - & - \\
\hline 6 & - & - & - & - & - & + & - & - & - \\
\hline 7 & + & + & + & + & - & - & + & + & - \\
\hline 8 & - & - & - & - & + & + & - & - & - \\
\hline 9 & + & + & - & - & + & - & - & + & - \\
\hline 10 & - & - & - & - & + & - & - & - & + \\
\hline
\end{tabular}


Table 3 Family characteristics

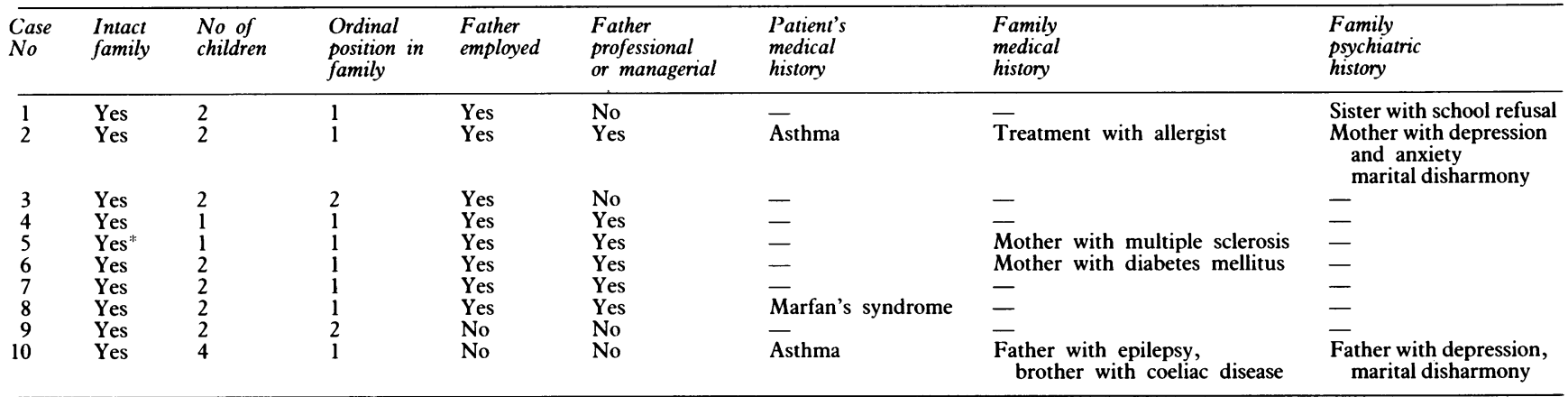

*Father had previous marriage.

ever, these were not given at a dosage or for a duration that would enable any conclusions about their effectiveness to be drawn. Those patients who had depressed mood were not consistently depressed and they were able to experience pleasure some of the time. None expressed feelings of hopelessness, worthlessness, a sense of guilt, or suicidal ideas.

Case 5 had specific fears as well as generalised anxiety. She had a long history of fear of needles and bats. She then developed considerable apprehension about normal hypnogogic images, and she experienced parts of her body as larger than normal, a symptom of anxiety known as depersonalisation.

Case 7, who developed a sudden paralysis of the lower limbs, had selective amnesia for the event. This was a dissociative phenomenon, that is a narrowing of the field of consciousness, a symptom associated with hysteria.

\section{ABSENCE FROM SCHOOL}

All of the patients had considerable absences from school (see table 1). For clarity where a patient missed over $50 \%$ of a school term they are considered to have been absent for that term. School absence ranged from half a term to two complete years.

Of the total series, six patients had serious exacerbations of symptoms (reported to be associated with a further viral illness, such as influenza) which occurred shortly after starting a new school term.

INDIVIDUAL CHARACTERISTICS

All of the patients were in the average range of intelligence and none had a history of developmental or learning difficulties, emotional or behavioural problems.

Cases 7 and 8 were noted to have significant difficulties with social relationships with peers. Case 7 was a shy conscientious girl who was 'old fashioned' in her tastes and attitudes and so she did not fit in with the groups at school. Case 8 has Marfan's syndrome and has been unusually tall since the age of 5 years. He felt self conscious and awkward and was teased at school.

FAMILIES

All of the 10 families were intact, that is to say that the biological parents were married and living together with the child (see table 3 ). In two families there were long standing marital difficulties. None of the families was from an ethnic minority.

\section{PSYCHIATRIC DIAGNOSIS}

Great difficulty is encountered when considering psychiatric diagnoses in these patients and no classification scheme is satisfactory. The descriptive term neurasthenia is useful in some cases, particularly case 4 . However, this is a term first used in the 19th century and it has been little used more recently. ${ }^{14} 15$

The consideration of depression is particularly difficult as a number of the symptoms characteristics of CFS - sleep disturbance, difficulty concentrating, and decreased energy-also occur in depressive illness. Although six of the 10 patients experienced depressed mood, none meet the Diagnostic and Statistical Manual of Mental Disorders Third Edition, Revised (DSM III-R) criteria for the depressive disorders. ${ }^{16}$ Similarly none of the patients meet criteria for the specific anxiety disorders. The most useful diagnostic category is somatisation disorder except that many of the criteria are not appropriate to children and adolescents.

\section{Management and the active rehabilitation programme}

After physical investigations excluded serious underlying organic pathology the patient and family were reassured, and an explanation of CFS was given recognising the suffering and distress of the patient. Specific psychiatric treatment was given where appropriate, for example cognitive therapy for anxiety in case 8, and dynamic and systemic issues were addressed in individual and family interviews.

An active rehabilitation programme was offered to all cases. This is a behavioural programme of gradual increase in the patients activities in a number of areas including school attendance, leisure, social, and physical activities. The patient and a parent were seen once a week with the aim of rehabilitation in one to three months. Baseline information was first obtained to determine the current level of activity. This information was obtained at interview and with a diary kept by the patient listing all activities and rest periods; this gives valuable details and can be used to measure 
progress if used throughout the rehabilitation. It is important that the patient has a sense of participation in the programme and they were involved in setting specific goals for increasing activity.

The programme moved from baseline activities in all areas in gradual stages. Direct contact with the school was essential to negotiate a gradual increase in participation in the school timetable. The patient was encouraged to go out for education every day and home tuition was avoided wherever possible. Prolonged illness can lead to social isolation and an increase in social and enjoyable activities provided more positive goals and increased motivation for school return. Physical activity was increased with exercises, outings, and a gradual reintroduction of sporting activities.

In cases where there were symptoms causing physical restriction or the patient had been housebound for some time, inpatient rehabilitation on a paediatric or other medical ward was offered. A similar approach was described by Dubowitz and Hersov in hysterical disorder. ${ }^{17}$ This was found to be the setting that was most acceptable to these patients and families, and it offered an enhancement of the programme by the involvement of other professional staff. Nurses, occupational therapists, and teachers were involved in increasing self care, lesiure, and academic activities. A physiotherapist provided exercises and special equipment, including hydrotherapy, to increase physical activity. A detailed active rehabilitation programme was drawn up by a member of the CPLT with specific targets agreed by the patient and the whole team. Pleasurable activities, such as trips out, acted as encouragement for goals reached in other areas. An inpatient admission of two to eight weeks was followed by weekly outpatient appointments to continue the programme until there was a return to normal functioning.

\section{Outcome}

Cases 3 and 8 showed gradual improvement with outpatient psychiatric treatment, which included individual and family interviews and a programme of active rehabilitation. At the end of six months case 3 was symptom free and engaging in normal activities. Case 8 was attending college full time at one year follow up. His physical symptoms were much improved but he continued to have some difficulties with social relationships. In case 10 the mother sought psychiatric advice when she noticed that her daughter's fatigue and misery were greatly alleviated during periods spent away from the family. Once the focus was diverted from the patient to the chronic severe marital difficulties her symptoms quickly subsided.

The four patients with symptoms causing physical restriction were offered inpatient treatment. Case 7 made a dramatic recovery when on the third day of her admission the paralysis in her legs suddenly disappeared. After two weeks she was walking unaided and after three weeks doing headstands and fitness training. She remained an inpatient for one month. Eighteen months later she was symptom free and attending college full time.

Case 9 recovered more gradually and was an inpatient for two months but maintained her improvement and was symptom free at follow up six months later.

The occurrence of loss of motor function appeared to improve outcome in these two cases as it brought about a realisation on the part of the parents of the importance of psychological factors and these patients made a particularly good recovery. Case 1 was an inpatient for two weeks and improved considerably but she and her family were not convinced that there was no organic basis for her symptoms and they recurred soon afterwards.

Three of the families (cases 4, 5, and 6) failed to attend a second outpatient psychiatric appointment and case 2 refused inpatient treatment. In these cases the parents were convinced that their child's illness was purely physical in origin and that active rehabilitation would be harmful.

Where the patient and parents accepted treatment, including an active rehabilitation programme, a substantial improvement was achieved in every case.

\section{Discussion}

This series illustrates that the children and adolescents who have the symptoms of CFS are an important group upon which the medical profession should focus attention. They may develop prolonged and severely debilitating symptoms, the nature of which may be difficult to interpret as many of the symptoms also occur in psychiatric disorders.

Cartesian dualism, that is the separation of the body and the mind, may be the origin of some of the confusion and controversy surrounding this subject. These cases suggest that the most useful model regarding CFS is a complex interaction of physical and psychological components. A valuable hypothesis is that in the minority of cases where there is a prolonged course or an associated psychiatric disorder that the experience of fatigue is maintained by other psychosocial factors. These psychosocial factors include individual and family factors and wider issues of clinical management and expectation of continuing activity and school attendance.

Possible individual maintaining factors are earlier experience of prolonged illness, poor social adjustment, problems at school, or high expectations of achievement before the illness.

The role of depression in CFS is uncertain. In a study by Wessely and Powell $47 \%$ of adult patients with CFS also fulfilled internationally accepted diagnostic criteria for affective disorder, and $25 \%$ had other psychiatric diagnoses including somatisation disorder, phobic and generalised anxiety disorders, and conversion disorder. ${ }^{11}$ Childhood emotional disorders are less well differentiated than adult disorders, however associated psychiatric disorder may act as a maintaining factor in CFS.

Two relevant concepts in psychiatry illustrate the complex interactions of the physical and 
psychological. The first is abnormal illness behaviour, ${ }^{18}$ which encompasses the range of psychiatric diagnoses that have been given to patients with physical complaints for which no adequate organic cause can be found. These include hypochondriasis, neurasthenia, conversion hysteria, and somatisation disorders. Goodyer has shown that hysterical conversion in childhood frequently involves disturbance of gait and lower limb function. ${ }^{19}$ Abnormal illness behaviour can occur without physical illness, it may be a prolongation of symptoms originally part of an organically determined disease, or it may accompany an undoubted organic disease. It is as Taylor describes when discussing hysteria, in a different domain from physical illness and it in no way invalidates it. ${ }^{20}$ The cases where there was loss of motor function suggest that abnormal illness behaviour can be a very important mechanism in CFS.

School refusal is the second relevant psychiatric concept. This is where the child is reluctant to go to school and remains at home. In many cases there is anxiety about separation on the part of the mother as well as the child. School refusal may follow a period of physical illness. A variant has been described 'the masquerade syndrome' by Waller and Eisenberg. ${ }^{21}$ This is school refusal disguised as physical illness. Parents can become intensely involved in the idea of their child's illness, making it impossible for him to go to school. The close connection, in some of our cases, of exacerbations of symptoms with the beginning of a new school term suggests that the mechanisms encountered in school refusal may play an important part.

Family factors are particularly important in the development of psychological disturbance in children and adolescents. Physical and psychiatric illness in other members of the family or marital problems may be important in maintaining illness on the part of the child. The intact professional or managerial high achieving family may increase the risk of a child developing severe or prolonged disabilities. The majority of our patients were from this type of family where they were the elder of one or two children. This may be interpreted as a referral bias. However, one can speculate that these families have a high investment in their children and that there is pressure to achieve on either the part of the parent or the child. There may then be an inability to accept reduction of energy and performance after illness. These families may also have difficulty in negotiating the issues of growing independence in adolescence, which would be particularly relevant with an elder female child.

Parental beliefs appear to be of vital importance in CFS. These families frequently hold firm beliefs in the purely physical nature of their child's difficulties. Families are often exposed to the literature of the ME [Myalgic encephalomyelitis] Association and the Action Campaign. These associations give a view of a single disease entity with a purely viral cause, where physical exertion may be harmful and must be avoided, and where rest is the recommended treatment. Rest may act in a circular way so that after rest when physical exertion is attempted normal physiological sensations are interpreted as a relapse. The beliefs of a parent can be so prominent that 'myalgic encephalomyelitis by proxy' possibly a variant of 'Münchausen syndrome by proxy' has been described. ${ }^{22}$

The individual should be seen in the context of his psychosocial predicament. ${ }^{20}$ As well as individual and family factors this includes wider social influences. Continuing medical investigation or contradictory recommendations from the medical profession, who often find themselves unable to offer effective solutions, may be maintaining factors. Parents may become very involved in their child's illness and this may even inadvertently prolong or exacerbate the situation. The families may turn to 'alternative' treatments where their experience of illness is validated and complex treatments including restricted diets and numerous vitamin and mineral supplements may be recommended. Home tuition may also validate illness behaviour and should be avoided except as part of a rehabilitation programme.

In conclusion it is proposed that the combined approach of a paediatrician and a child psychiatrist is adopted. This is of particular value in the difficult process of engaging the patient and family in treatment. In those cases where treatment was refused or of limited success, there was a continuing belief in a purely organic basis to the symptoms. It is important that psychiatric referral is not delayed in those patients where there are significant emotional factors, failure of progress in treatment, or a prolonged course. An active rehabilitation programme, in conjunction with specific psychiatric treatment where appropriate, when accepted is an effective treatment approach.

There is a need for systematic research into this fascinating condition in childhood and adolescence for the establishment of internationally accepted criteria, data on incidence, and outcome studies. Criteria for adults, Holmes et al for example, ${ }^{3}$ require symptoms for at least six months. This is almost certainly too long for children where a period of two months would be more appropriate.

Childhood and adolescence is a time of enormous physical, emotional, and social changes with considerable implications for personality development. These children are in danger of profound interference with normal psychological development and chronic dysfunction. They must not be left in a no man's land between paediatrics and psychiatry.

I thank Dr Pieter van Boxel and Penny Spinks of the Child Psychiatry Liaison Team, Paxton House; and the paediatric department of the Royal Berkshire and Battle Hospitals, Reading.

1 McEvedy CP, Beard AW. Royal Free Epidemic of 1955: a reconsideration. $B M \mathcal{F} 1970 ; 1: 7-11$.

2 David AS, Wessely S, Pelosi AJ. Postviral fatigue syndrome: time for a new approach. $B M \mathcal{A}$ 1988;296:696-9.

3 Holmes GP, Kaplan JE, Gantz NM, et al. Chronic fatigue syndrome: a working case defintion. Ann Intern Med 1988;108:387-9.

4 Swartz MN. The chronic fatigue syndrome: one entity or many? N Engl 7 Med 1988;319:1726-8.

5 Yousef GE, Mowbray JF, Mann GF, et al. Chronic entero- 
virus infection in patients with post viral fatigue syn drome. Lancet 1988;i:146-50.

6 Fegan KG, Behan PO, Bell EJ. Myalgic encephalomyelitis: report of an epidemic. $\mathcal{F} R$ Coll Gen Pract 1983:33:335-7.

7 Tobi M, Morag A, Ravid Z. Prolonged atypical illness associated with serological evidence of persistent EpsteinBarr virus infection. Lancet 1982;i:61-4.

8 Jones JF, Ray CG, Minnich LL, Hicks MJ, Kibler R, Lucas DO. Evidence for active Epstein-Barr virus infection in patients with persistent, unexplained illnesses: elevated anti-early antigen antibodies. Ann Intern Med 1985;102: anti-7.

9 Gold D, Bowden R, Sixbey J, et al. Chronic fatigue. A prospective clinical and virologic study. $\mathcal{F} A M A$ 1990;264: prospecti

10 Bowman SJ, Brostoff J, Newman S, Mowbray JF. Postviral syndrome-how can a diagnosis be made? A study of patients undergoing a Monospot test. $\mathcal{F} R$ Soc Med $1989 ; 82: 712-6$

11 Wessely S, Powell R. Fatigue syndromes: a comparison of chronic 'post-viral' fatigue with neuromuscular and affective disorders. I Neurol Neurosurg Psychiatry 1989;52:940-8.

12 Lask B, Dillon MJ. Postviral fatigue syndrome. Arch Dis Child 1990;65:1198.
13 Harris F, Taitz LS. Damaging diagnoses of myalgic encephalitis in children. $B M \mathcal{F} 1989 ; 299: 790$

14 White P. Fatigue syndrome: neurasthenia revisited. $B M \mathcal{Y}$ 1989;298:1199-200.

15 Wessely $S$. Old wine in new bottles: neurasthenia and ' $M E$ '. Psychol Med 1990;20:35-53.

16 American Psychiatric Association. Diagnostic and statistical manual of mental disorders. Revised. 3rd Ed. Washington DC: APA, 1987.

17 Dubowitz V, Hersov L. Management of children with nonorganic (hysterical) disorders of motor function. Dev Med Child Neurol 1976;18:358-68.

18 Pilowsky I. Abnormal illness behaviour. Br 7 Med Psychol 1969;42:347-51.

19 Goodyer I Hysterical conversion reactions in childhood. f Child Psychol Psyhiatry 1981;22:179-88.

20 Taylor DC. Hysteria, play-acting and courage. Br 7 Psychiatry 1986;149:37-41.

21 Waller D, Eisenberg L. School refusal in childhood: psychiatric/paediatric perspective. In: Hersov L, Berg I eds. Out of school: modern perspectives in school refusal and truancy. Chichester: Wiley, 1980:209-29.

22 MacDonald TM. Myalgic encephalomyelitis by proxy. $B M \gamma$ 1989;299:1030.

\section{Prolactin and seizures}

I've heard of post hoc ergo procter hoc but never before of pre hoc ergo procter hoc. To the best of my knowledge the use of serum prolactin measurements to distinguish between true and false fits was first described by Michael Trimble in the British Medical Fournal in $1978 .^{1}$ A recent report from Israel (Nathanel Zelnick and colleagues, Pediatrics $1991 ; 88: 486-9$ ) is the latest of several to confirm that a rise in serum prolactin concentration occurs after generalised tonic-clonic seizures and not after febrile convulsions, hysterical attacks, breath holding, or syncope. After complex partial seizures there may be a lesser and less reliable rise in serum prolactin and with other kinds of seizure the test is not helpful. ${ }^{2}$ The Israeli workers refer to several papers published between 1980 and 1987 and say that they led to Trimble and others suggesting that the phenomenon could be helpful in distinguishing between epilepsy and non-epilepsy.

Does precedence matter? In the ultimate analysis I suppose not but it's as well to get things right if only to keep the historical record straight.

ARCHIVIST

1 Trimble MR. Serum prolactin in epilepsy and hysteria. $B M \mathcal{F}$ 1978;ii: 1682

2 Bye AME, Nunn KP, Wilson J. Prolactin and seizure activity. Arch Dis Child 1985;60:848-51. 